\title{
INPUT/OUTPUT SELECTION FOR ROBUST CONTROL
}

\author{
Marc van de Wal and Bram de Jager \\ Faculty of Mechanical Engineering, Eindhoven University of Technology \\ P.O. Box 513, 5600 MB Eindhoven, The Netherlands
}

\begin{abstract}
Two new Input/Output (IO) selection methods are proposed. The aim is to find the IO sets for which there exists a controller that achieves robust performance. IO selection could thus be based on $\mu$ synthesis, but to avoid this often time-consuming process $\mathcal{H}_{\infty}$ controller existence conditions are used instead. To reduce conservatism, the structure in the combined uncertainty/ performance block $\Delta$ is "eliminated." The first (sufficiency-based) method augments the plant by diagonal scalings, whereas the second (necessity-based) method absorbs an uncertainty representation into the plant. An active suspension control problem illustrates both methods.
\end{abstract}

Key words: robust control, linear systems, actuator and sensor selection.

\section{Introduction}

Prior to controller design, suitable manipulated inputs (actuators) and measured outputs (sensors) must be selected. This Input/Output (IO) selection process is crucial, since the employed IO set affects the achievable performance, as well as reliability and the costs of hardware, operation, and maintenance. Since the number of candidate IO sets may be large, a systematic IO selection method would be welcome to complement physical insight and to avoid that favorable IO sets are overlooked. For a quick evaluation of IO sets, such a method should be efficient. It should also be effective, in the sense that only those 10 sets are accepted (or eliminated) for which the intended objective can (or cannot) be met.

This paper considers linear control systems. The IO selection goal is to find the 10 sets for which there exists a controller achieving Robust Performance (RP), i.e., guaranteed performance for a class of uncertainties. Such IO sets are termed viable. The proposal of two new IO selection methods aimed at this goal is our main contribution.

In the "standard" control system set-up, RP is accounted for by a combined uncertainty/performance block $\Delta$. Due to the structure of $\Delta$, IO selection based on $\mu$-synthesis may be an effective but time- consuming approach. If $\Delta$ were not structured (as for nominal performance), IO selection based on checking $\mathcal{H}_{\infty}$ controller existence conditions would be less demanding [4]. This gives rise to the basic idea of "eliminating" the structure of $\Delta$.

The firstly proposed method employs diagonal plant scalings to account for the structure in $\Delta$. This results in a sufficient condition for RP and IO sets may be incorrectly rejected. The secondly proposed method replaces the (possibly structured) uncertainty block by a suitable uncertainty representation, which is then absorbed into the plant and the unstructured performance block remains. A necessary condition for the achievement of RP results and IO sets may be incorrectly accepted. The two methods can be combined to improve effectiveness. Both are applied to an active suspension control problem for a tractor-semitrailer.

\section{Robust performance}

In the following, $\mathcal{F}_{l}(A, B)$ and $\mathcal{F}_{u}(A, B)$ denote lower and upper linear fractional transformations [7, Chapter 10] (" $A$ closed by $B$ "). Finite-dimensional, linear, time-invariant control systems in the set-up of Fig. 1 are considered. $G$ is the generalized plant including the plant model and design filters quantifying control objectives and uncertainties, $K$ is the controller, and $M=\mathcal{F}_{l}(G, K)$ is the generalized closed-loop system. The block $\Delta_{u}$ extracts the uncertainties from the plant and the fictitious block $\Delta_{p}$ accounts for performance requirements. The measured variables (outputs) are denoted by $y$, the manipulated variables (inputs) by $u$, the regulated variables by $z_{p}$, the exogenous variables (references, disturbances, noise) by $w_{p}$, and the uncertainty-related variables by $w_{u}$ and $z_{u}$. Finally, $w:=\left(\begin{array}{l}w_{u} \\ w_{p}\end{array}\right)$ and $z:=\left(\begin{array}{l}z_{u} \\ z_{p}\end{array}\right)$.

The following set of uncertainties is assumed:

$$
\Delta_{u}:=\left\{\Delta_{u}=\operatorname{diag}\left(\Delta_{u_{1}}, \ldots, \Delta_{u_{k}}\right): \Delta_{u_{i}} \in \mathcal{R} \mathcal{H}_{\infty}\right\} .
$$

$\mathcal{R} \mathcal{H}_{\infty}$ denotes real-rational, proper, and stable Transfer Function Matrices (TFM's) and $\boldsymbol{\Delta}_{\boldsymbol{u}}^{\boldsymbol{\beta}}$ will denote the subset of $\Delta_{u}$ with $\left\|\Delta_{u}\right\|_{\infty} \leq \beta$. Whereas the exclusion of repeated dynamic blocks is only for notational convenience, the exclusion of real parametric 


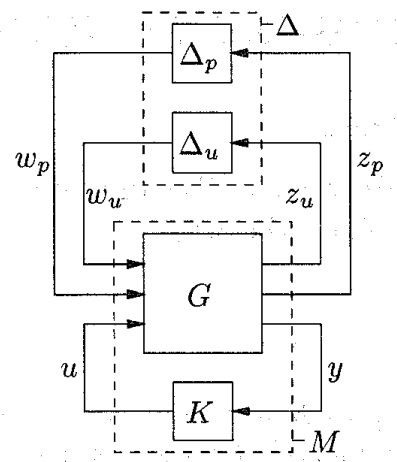

Figure 1: Standard control system set-up

blocks is restrictive. Extensions to mixed parametric/dynamic uncertainties may be possible, but to avoid complications in controller design and IO selection, real parametric uncertainties are covered by dynamic ones. This may be conservative. The combined uncertainty/performance block is defined as $\Delta:=\operatorname{diag}\left(\Delta_{u}, \Delta_{p}\right)$.

As a measure of RP, the $\mathcal{H}_{\infty}$ norm of the uncertain TFM $\mathcal{F}_{u}\left(M, \Delta_{u}\right)$ between $w_{p}$ and $z_{p}$ is used. The following provides a necessary and sufficient condition for RP [7, Chapter 11]:

Robust Performance: Let $\gamma>0$ and let $\Delta_{u}$ and $M$ be stable. For all $\Delta_{u} \in \Delta_{u}^{\mathbf{1} / \gamma}$, the uncertain system $\mathcal{F}_{u}\left(M, \Delta_{u}\right)$ is stable and $\left\|\mathcal{F}_{u}\left(M, \Delta_{u}\right)\right\|_{\infty}<\gamma$, i.e., $\mathrm{RP}$ is achieved for all $\Delta_{u} \in \Delta_{u}^{1 / \gamma}$, if and only if:

$$
\|M\|_{\mu}<\gamma, \text { with }\|M\|_{\mu}:=\sup _{\omega} \mu_{\Delta}(M(j \omega)) .
$$

Here, $\mu$ is the structured singular value. For a complex matrix $M$ and a block structure $\Delta, \mu$ is defined as:

$$
\mu_{\Delta}(M):=\frac{1}{\min _{\Delta \in \Delta}(\bar{\sigma}(\Delta): \operatorname{det}(I-M \Delta)=0)}
$$

unless no $\Delta \in \Delta$ makes $I-M \Delta$ singular, in which case $\mu_{\Delta}(M):=0$. For general $\Delta$, exact computation of $\mu$ is $\mathcal{N} \mathcal{P}$ hard. As a tractable approximation, lower and upper bounds are used in practice. The lower bound is only of indirect interest for the IO selection methods and not defined here. Throughout this paper it is assumed that the lower bound exactly equals $\mu$. The upper bound is defined as:

$$
\mu_{u}(M):=\min _{D \in \mathcal{D}} \vec{\sigma}\left(D_{z} M D_{w}^{-1}\right) .
$$

In analogy to (2), $\|M\|_{\mu_{u}}:=\sup _{\omega} \mu_{u}(M(j \omega))$. In (4), $D \in \mathcal{D}$ is shorthand for $D_{z} \in \mathcal{D}_{z}, D_{w} \in \mathcal{D}_{w}$, with $D_{z}$ and $D_{w}$ the " $D$-scales" in the sets:

$$
\begin{aligned}
& \mathcal{D}_{z}:=\left\{\operatorname{diag}\left(d_{1} I_{m_{1}}, \ldots, d_{l} I_{m_{l}}\right): d_{j} \in \mathbb{R}^{+}\right\}, \\
& \mathcal{D}_{w}:=\left\{\operatorname{diag}\left(d_{1} I_{n_{1}}, \ldots, d_{l} I_{n_{l}}\right): d_{j} \in \mathbb{R}^{+}\right\} .
\end{aligned}
$$

Each $n_{i} \times m_{i}$ full block $\Delta_{u_{i}}$ in $\Delta_{u}$ is accompanied by diagonal scaling matrices $d_{i} I_{m_{i}}$ and $d_{i} I_{n_{i}}$. The $D$ scales are normalized with respect to the last diagonal matrix corresponding to $\Delta_{p}: d_{l}=1$. Since $D_{z}$ and $D_{w}$ only differ in dimension, " $D$ " will be used as a brief notation for " $D_{z}$ and $D_{w}$."

\section{The IO selection procedure}

An IO selection method based on RP checks the existence of a stabilizing controller $K$ making $\|M\|_{\mu}<\gamma$. An effective but time-consuming method would check this by $\mu$-synthesis, e.g., by $D-K$ iteration [7, Chapter 11]. If $\Delta$ were unstructured, $\|M\|_{\mu}=\|M\|_{\infty}:=$ $\sup _{\omega}(M(j \omega))$ and computationally less demanding $\mathcal{H}_{\infty}$ controller existence conditions (Section 5) could be used. The basic idea of the new IO selection methods is therefore to get rid of the structure in $\Delta$ (Section 4). The four main steps of the two new methods are the same and listed below. The superscript $\{\cdot\}^{\star}$ to $M$ will be used to denote the closed-loop system for the full IO set, i.e., the IO set including all candidate inputs and outputs.

1. Perform $D-K$ iteration for the full IO set and assume convergence to the global optimum $\left\|M^{\star}\right\|_{\mu}$.

2. Based on the full IO set's optimal $M$, construct the $D$-Scale Estimate (DSE) $\tilde{D}(s)$ or the WorstCase Uncertainty (WCU) $\tilde{\Delta}_{u}(s)$.

3. For the other IO sets, the generalized plants are modified according to:

$$
\begin{aligned}
& G_{\mathrm{DSE}}=\left[\begin{array}{cc}
\tilde{D}_{z} & 0 \\
0 & I
\end{array}\right] G\left[\begin{array}{cc}
\tilde{D}_{w}^{-1} & 0 \\
0 & I
\end{array}\right], \\
& G_{\mathrm{WCU}}=\mathcal{F}_{u}\left(G, \tilde{\Delta}_{u}\right) .
\end{aligned}
$$

For $u$ and $y$ that are not considered, the entries in $\tilde{D}$ corresponding to input weights (in $z_{p}$ ) and output noise (in $w_{p}$ ) must be skipped, as well as the entries in $\tilde{D}$ and $\tilde{\Delta}_{u}$ corresponding to input and output uncertainty (in $z_{u}$ and $w_{u}$ ).

4. Use $\mathcal{H}_{\infty}$ controller existence conditions to check if there is a stabilizing $K$ achieving $\left\|\mathcal{F}_{l}\left(G_{\mathrm{DSE}}, K\right)\right\|_{\infty}<\gamma$ or $\left\|\mathcal{F}_{l}\left(G_{\mathrm{WCU}}, K\right)\right\|_{\infty}<\gamma$.

Hence, $D-K$ iteration is only performed for the full IO set, whereas for the other IO sets computationally less demanding operations are performed. To further improve efficiency, subsets of larger nonviable IO sets are not checked, since eliminating actuators or sensors will not improve the best achievable performance (for the same reason, IO selection with $\gamma<\left\|M^{\star}\right\|_{\mu}$ is useless). More sophisticated strategies for going through the IO sets are currently investigated.

\section{Elimination of structure}

This section discusses the construction of a DSE and a WCU to be used in Step 2 of the IO selection methods. For this purpose, a frequency grid is employed that is assumed to cover all relevant frequencies.

\section{$4.1 \quad D$-scale estimate}

For a given $M$, the convex optimization problem (4) can be solved frequency-by-frequency, e.g., 
with the $\mu$-Tools function mu. This returns the $D$ scale data $D(j \omega)$. To account for the structure of $\Delta$ during IO selection, a $D$-Scale Estimate (DSE) $\tilde{D}(s), \tilde{D}^{-1}(s) \in \mathcal{R} \mathcal{H}_{\infty}$ approximating this data is constructed (a similar approximation is performed during $D-K$ iteration). The DSE can be constructed with the $\mu$-Tools function msf. For given $M(j \omega)$, $D(j \omega)$, and $\tilde{D}(j \omega)$, the following inequality holds:

$$
\mu_{\Delta}(M) \leq \bar{\sigma}\left(D_{z} M D_{w}^{-1}\right) \leq \bar{\sigma}\left(\tilde{D}_{z} M \tilde{D}_{w}^{-1}\right) .
$$

The higher the order of the DSE, the tighter the right inequality may be. However, $G$ is augmented by $\tilde{D}_{z}$ and $\tilde{D}_{w}^{-1}(6)$. So, increasing the order of the DSE increases the order of the modified plant and the computation time for IO selection. Qualitatively, the order of the DSE is sufficiently large if the difference between $\bar{\sigma}\left(D_{z} M^{\star} D_{w}^{-1}\right)$ and $\bar{\sigma}\left(\tilde{D}_{z} M^{\star} \tilde{D}_{w}^{-1}\right)$ is "small." For a possible quantification, see [1, Chapter 5].

$\tilde{D}$ for the full IO set's optimal closed-loop $M^{\star}$ is used to augment the generalized plants for other IO sets. The DSE method exhibits a sufficient character, because for a given DSE $\tilde{D}$ and corresponding $G_{\mathrm{DSE}}$, the following holds [5]:

$\min _{K}\left\|\mathcal{F}_{l}(G, K)\right\|_{\mu}<\gamma \Leftarrow \min _{K}\left\|\mathcal{F}_{l}\left(G_{\mathrm{DSE}}, K\right)\right\|_{\infty}<\gamma$.

For the full IO set, this implication turns into an equivalence if $\tilde{D}$ perfectly matches the DSE data for the optimal $M^{\star}$ and if $\mu=\mu_{u}$.

The sufficiency is caused by the following three sources, in increasing order of importance. The first source is due to the $D$-scales only giving an upper bound for $\mu$. The second source is the imperfectness of $\tilde{D}$ for the full IO set's DSE data. The third source is the use of a fixed $\tilde{D}$ for other IO sets than the full one, whereas a combined optimization over $K$ and $\tilde{D}$ (like in $D-K$ iteration) generally yields results closer to $\|M\|_{\mu}$. Due to sufficiency, the DSE method may incorrectly eliminate IO sets, see Section 6.

The motivation to, nevertheless, use $\tilde{D}$ from the full IO set also for the other IO sets is based on the following heuristic. The DSE's $\tilde{D}_{z}$ and $\tilde{D}_{w}$ from a particular IO set's optimal closed-loop $M_{1}$ may also be "representative" for another IO set's optimal closed-loop $M_{2}$, in the sense that $\bar{\sigma}\left(\tilde{D}_{z} M_{1} \tilde{D}_{w}^{-1}\right) \approx \bar{\sigma}\left(\tilde{D}_{z} M_{2} \tilde{D}_{w}^{-1}\right)$. The DSE's from the full IO set may now be representative for other IO sets which are equally good: such IO sets may have almost the same $M$ and $\mu_{\Delta}(M)$ as the full IO set and, if so, they have almost the same $D$-scales. Hopefully, using $\tilde{D}$ from the full IO set yields better results than using no scaling at all $(\tilde{D}=I)$, but this is not guaranteed.

\subsection{Worst-case uncertainty}

For a given $M$ with $\mu_{\Delta}(M)>0$, the definition of $\mu$ (3) shows that there is a $\Delta$ with $\bar{\sigma}(\Delta)=1 / \mu_{\Delta}(M)$ which makes $I-M \Delta$ singular. A $\Delta$ which does so across frequency, denoted by $\bar{\Delta}$, could be constructed with the $\mu$-Tools function mu. The part of $\bar{\Delta}$ corresponding to $\Delta_{u}$ is called the Worst-Case Uncertainty (WCU) data and denoted by $\bar{\Delta}_{u}$. With $M_{u u}$ corresponding to the loop around $\Delta_{u}$, an essential assumption now is that $\mu_{\Delta_{u}}\left(M_{u u}\right) \leq \mu_{\Delta}(M)$. This is often justified in practice, since it means that the robust stability problem is easier than the RP problem. For $M$ with $\|M\|_{\mu}=\alpha$, there is a $\Delta_{u} \in \Delta_{u}^{1 / \alpha}$ that violates RP, i.e., that causes $\left\|\mathcal{F}_{u}\left(M, \Delta_{u}\right)\right\|_{\infty}=\alpha$. Such a TFM, denoted by $\hat{\Delta}_{u}(s)$, is called a WCU.

To eliminate the structure of $\Delta$ during IO selection, a suitable WCU must be constructed. This may not be straightforward for two reasons. First, $\hat{\Delta}_{u}$ must be bounded by $\frac{1}{\alpha}=\inf _{\omega} \bar{\sigma}\left(\bar{\Delta}_{u}\right)$. So, the magnitudes of $\hat{\Delta}_{u}$ and $\bar{\Delta}_{u}$ may differ significantly. Second, even if $\bar{\sigma}\left(\bar{\Delta}_{u}\right)$ were flat across frequency $\left(\bar{\sigma}\left(\bar{\Delta}_{u}\right)=\frac{1}{\alpha}\right)$, it would in general be impossible to construct a stable and low-order TFM which well approximates $\bar{\Delta}_{u}$ in both magnitude and phase [3] (it would be possible for a stable but high-order TFM, or a loworder but unstable TFM, which are not practical and not allowed, respectively). For given $M(j \omega)$ with $\|M\|_{\mu}=\alpha$ and $\hat{\Delta}_{u}(j \omega)$ with $\bar{\sigma}\left(\hat{\Delta}_{u}(j \omega)\right) \leq \frac{1}{\alpha}$, the following inequality holds:

$$
\mu_{\Delta}(M)=\bar{\sigma}\left(\mathcal{F}_{u}\left(M, \bar{\Delta}_{u}\right)\right) \geq \bar{\sigma}\left(\mathcal{F}_{u}\left(M, \hat{\Delta}_{u}\right)\right) .
$$

The size of the gap depends on the mismatch between $\hat{\Delta}_{u}$ and $\bar{\Delta}_{u}$. The construction by the $\mu$-Tools function dypert is only based on the WCU data $\bar{\Delta}_{u}(j \bar{\omega})$, with $\bar{\omega}$ the frequency where $\mu_{\Delta}(M)$ has its supremum. As a result, the WCU is perfect for $\bar{\omega}$ and (10) becomes an equality, but it nay be bad for other $\omega$. Each block $\hat{\Delta}_{u_{i}}$ is all-pass, i.e., $\bar{\sigma}\left(\hat{\Delta}_{u_{i}}(j \omega)\right)=\frac{1}{\alpha}$. Alternative construction procedures were proposed in [3], but the WCU by dypert appeared to work best and is adopted in this paper.

$\hat{\Delta}_{u}$ for the full IO set's optimal closed-loop $M^{\star}$ is absorbed into the generalized plants for other IO sets. In this way, the unstructured performance block $\Delta_{p}$ remains. If $\alpha^{\star}:=\left\|M^{\star}\right\|_{\mu}$ is smaller than the requirement $\gamma, \hat{\Delta}_{u}$ is outside the class of uncertainties against which RP is required. To resolve this, $\hat{\Delta}_{u}$ is scaled down to $\tilde{\Delta}_{u}=\frac{\alpha^{*}}{\gamma} \cdot \hat{\Delta}_{u} \in \Delta_{u}^{1 / \gamma}$ and $\tilde{\Delta}_{u}$ is used in Step 3 of IO selection. The WCU method has a necessary character, because for a given WCU $\tilde{\Delta}_{u}$ and the corresponding $G_{\mathrm{wCU}}$, the following holds [3]: $\min _{K}\left\|\mathcal{F}_{l}(G, K)\right\|_{\mu}<\gamma \Rightarrow \min _{K}\left\|\mathcal{F}_{l}\left(G_{\mathrm{WCU}}, K\right)\right\|_{\infty}<\gamma$.

For the full IO set, this implication turns into an equivalence if $\tilde{\Delta}_{u}=\hat{\Delta}_{u}$ perfectly matches the WCU data for the optimal $M^{\star}$. However, as argued above, such a construction is in general not practical.

The necessity is caused by the following two sources, in increasing order of importance. First, even for the full IO set itself, the difference between $\hat{\Delta}_{u}$ and the data $\bar{\Delta}_{u}$ may be large. In particular, for the dypert construction $\hat{\Delta}_{u}$ may not be worst-case for other fre- 
quencies than $\bar{\omega}$. The possible mismatch between the data and the forthcoming TFM is in general more serious for the WCU method than for the DSE method. The second source is due to the fact that the WCU from the full IO set may not be representative for other IO sets, i.e., the gap between $\bar{\sigma}\left(\mathcal{F}_{u}\left(M^{\star}, \hat{\Delta}_{u}\right)\right)$ and $\bar{\sigma}\left(\mathcal{F}_{u}\left(M, \hat{\Delta}_{u}\right)\right)$ may be large. Due to necessity, the WCU method may incorrectly accept IO sets, see Section 6. The reason why the WCU from the full IO set is still used for other IO sets is based on a similar heuristic as for the DSE. Hopefully, this $\tilde{\Delta}_{u}$ works better than no uncertainty at all.

\section{The selection conditions}

Step 4 of the IO selection procedure is discussed. The DSE and WCU attempt to account for the structure in $\Delta$. Conditions for the existence of a stabilizing controller achieving the $\mathcal{H}_{\infty}$ norm requirement $\|M\|_{\infty}<\gamma$ will now be used for IO selection. These conditions involve Algebraic Riccati Equations (ARE's). Four assumptions must then be satisfied on the open-loop TFM's from $u$ to $z$ and from $w$ to $y[7$, Chapter 17]. If necessary, the practical control problem could be slightly adapted to meet these assumptions. If conditions involving linear matrix inequalities [2] were used instead of ARE's, no assumptions would be imposed. The conditions employed here are given a short interpretation below (for details, see [7, Chapter 16\&17]). For notational convenience, $w, z$, and $G$ related to the original plant are used (Fig. 1), but the conditions apply for the modified plants $G_{\mathrm{DSE}}$ or $G_{\mathrm{WCU}}$ :

1. Check if the plant is stabilizable by $u$ and detectable from $y$.

2. Check if the open-loop direct feed-through from $w$ to $z$ is not "too large," since $K$ cannot completely cancel this effect.

3. Check if $K$ with full information on $w$ and the states of $G$ (ideal output set) meets $\|M\|_{\infty}<\gamma$.

4. Check if $K$ with full access to $z$ and the states of $G$ (ideal input set) meets $\|M\|_{\infty}<\gamma$.

5. Based on results from 3 and 4 , check if the combination of input set and output set meets $\|M\|_{\infty}<\gamma$.

As soon as one condition fails for a given IO set, the other conditions need not be checked.

\section{Active suspension example}

The IO selection methods are evaluated for an active suspension applied to the vehicle model in Fig. 2. Only a brief description of the control problem is given here. More details, including the design filters in $G$, are given in [5]. The RP goal is keeping

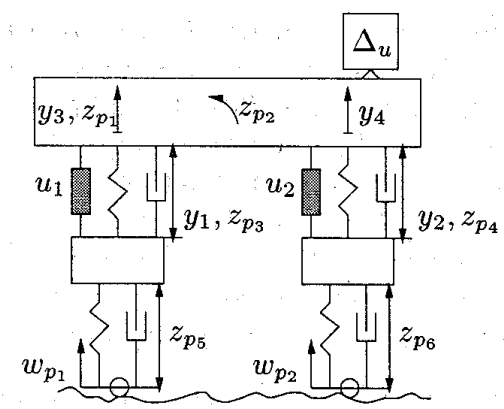

Figure 2: Tractor-semitrailer model

the accelerations $\left(z_{p_{1}}, z_{p_{2}}\right)$, the suspension deflections $\left(z_{p_{3}}, z_{p_{4}}\right)$, the tire deflections $\left(z_{p_{5}}, z_{p_{6}}\right)$, and the inputs $(u)$ small, subject to road irregularities $\left(w_{p_{1}}, w_{p_{2}}\right)$, sensor noise, and $90 \%$ semitrailer mass uncertainty. Though $\Delta_{u}$ is parametric, it must be treated as dynamic. $\Delta_{u}$ is a scalar and so $\tilde{\Delta}_{u}$ and $\tilde{D}$ are found by constructing scalar transfer functions. Two force actuators, two displacement sensors $\left(y_{1}, y_{2}\right)$, and two acceleration sensors $\left(y_{3}, y_{4}\right)$ are suggested. This yields the relatively small number of 45 candidate IO sets, so checking the IO selection results by $D-K$ iteration (DKI) becomes feasible.

\subsection{Results for typical IO sets}

Prior to IO selection, the potential ineffectiveness of the new methods is studied in some detail. Nine typical IO sets are subjected to "optimal" DKI, i.e., DKI till $\|M\|_{\mu}$ does not further converge, and $\mathcal{H}_{\infty}$ optimizations for the (modified) generalized plants. These IO sets give a good indication of the importance of each candidate actuator and sensor. The results are printed in Table 1 . The subscripts to $M$ specify the considered situation. Based on the $\left\|M_{\mathrm{RP}}\right\|_{\mu}$ values, it is concluded that the actuator $\left(u_{2}\right)$ and the sensors $\left(y_{2}\right.$ and $\left.y_{4}\right)$ at the rear of the tractor are more important for RP than those at the front.

\subsubsection{Results with DSE}

For the full IO set's optimal closed-loop $M_{\mathrm{RP}}^{\star}$, Fig. 3 compares the $D$-scale data and 3 rd and 4 th order DSE's. Figure 4 shows that the 4 th order DSE is better than the 3rd order DSE, in the sense that there is hardly any difference between $\bar{\sigma}\left(D_{z} M_{\mathrm{RP}}^{\star} D_{w}^{-1}\right)$ and $\bar{\sigma}\left(\tilde{D}_{z} M_{\mathrm{RP}}^{\star} \tilde{D}_{w}^{-1}\right)$.

For both the 3 rd and 4 th order DSE, $\mathcal{H}_{\infty}$ optimization results for $G_{\mathrm{DSE}}$ are listed in Table 1. For all IO sets, $\left\|M_{\mathrm{DSE}}\right\|_{\infty}$ for the 4 th order DSE is no larger than for the 3rd order DSE and closer to $\left\|M_{\mathrm{RP}}\right\|_{\mu}$. With the 4 th order DSE, IO sets with $\left\|M_{\mathrm{RP}}\right\|_{\mu}$ close to $\left\|M_{\mathrm{RP}}^{\star}\right\|_{\mu}=0.75$ (full IO set) also have $\left\|M_{\mathrm{DSE}}\right\|_{\infty}$ close to $\left\|M_{\mathrm{RP}}\right\|_{\mu}$. Note that computing $\left\|M_{\mathrm{RP} P}\right\|_{\infty}=\min _{K}\left\|\mathcal{F}_{l}(G, K)\right\|_{\infty}$ as if the combined uncertainty/performance block $\Delta$ were unstructured is the same as computing. $\left\|M_{\mathrm{DSE}}\right\|_{\infty}=$ 
Table 1: Nine IO sets with $\left\|M_{\mathrm{RP}}\right\|_{\mu}$ from optimal $D-K$ iterations, $\left\|M_{\mathrm{RP}}\right\|_{\infty}$ and $\left\|M_{\mathrm{NP}}\right\|_{\infty}$ from $\mathcal{H}_{\infty}$ optimizations with and without $\Delta_{u}$, and $\left\|M_{\mathrm{DSE}}\right\|_{\infty}$ and $\left\|M_{\mathrm{wCU}}\right\|_{\infty}$ from $\mathcal{H}_{\infty}$ optimizations with $\tilde{D}$ and $\tilde{\Delta}_{u}$.

\begin{tabular}{cccccccccccc}
\hline & IO set & $\left\|M_{\mathrm{RP}}\right\|_{\mu}$ & $\left\|M_{\mathrm{RP}}\right\|_{\infty}$ & \multicolumn{2}{c}{$\left\|M_{\mathrm{DSE}}\right\|_{\infty}$} & $\left\|M_{\mathrm{NP}}\right\|_{\infty}$ & \multicolumn{3}{c}{$\left\|M_{\mathrm{WCU}}\right\|_{\infty}$} \\
& & & & \multicolumn{3}{c}{ 3rd order } & 4th order & & $\gamma=1$ & $\gamma=0.8$ & $\gamma=\left\|M_{\mathrm{RP}}\right\|_{\mu}$ \\
\hline \hline $\mathbf{1}$ & $y_{1} y_{2} y_{3} y_{4}$ & $u_{1} u_{2}$ & 0.75 & 190 & 0.83 & 0.75 & 0.15 & 0.15 & 0.15 & 0.17 \\
2 & $y_{1} y_{2} y_{3} y_{4}$ & $u_{1}$ & 1.06 & 190 & 1.06 & 1.06 & 0.17 & 0.23 & 16.35 & 11.64 \\
3 & $y_{1} y_{2} y_{3} y_{4}$ & $u_{2}$ & 0.75 & 190 & 0.83 & 0.76 & 0.27 & 0.28 & 0.27 & 0.27 \\
4 & $y_{1} y_{2}$ & $u_{1} u_{2}$ & 0.76 & 436 & 0.83 & 0.77 & 0.15 & 0.15 & 0.19 & 0.25 \\
5 & $y_{3} y_{4}$ & $u_{1} u_{2}$ & 0.76 & 208 & 0.83 & 0.76 & 0.16 & 0.15 & 0.16 & 0.17 \\
6 & $y_{1}$ & $u_{1} u_{2}$ & 1.01 & 972 & 1.26 & 1.18 & 0.16 & 0.23 & 12.54 & 13.93 \\
7 & $y_{2}$ & $u_{1} u_{2}$ & 0.76 & 436 & 0.83 & 0.77 & 0.27 & 0.28 & 0.26 & 0.26 \\
8 & $y_{3}$ & $u_{1} u_{2}$ & 0.83 & 946 & 1.29 & 1.19 & 0.17 & 0.22 & 0.51 & 0.53 \\
$\mathbf{9}$ & $y_{4}$ & $u_{1} u_{2}$ & 0.76 & 288 & 0.83 & 0.77 & 0.28 & 0.28 & 0.27 & 0.27 \\
\hline
\end{tabular}

$\min _{K}\left\|\mathcal{F}_{l}\left(G_{\mathrm{DSE}}, K\right)\right\|_{\infty}$ for $\tilde{D}_{z}=I$ and $\tilde{D}_{w}=I$. The $\left\|M_{\mathrm{RP}}\right\|_{\infty}$ values clearly give a pessimistic indication of the achievable RP level. The DSE reasonably successfully accounts for the structure in $\Delta$, at least for this example.

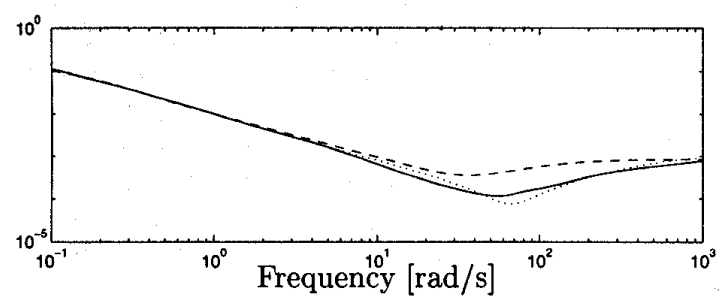

Figure 3: $D(-)$ and 3 rd $(--)$ and 4 th order $(\cdot \cdot) \tilde{D}$

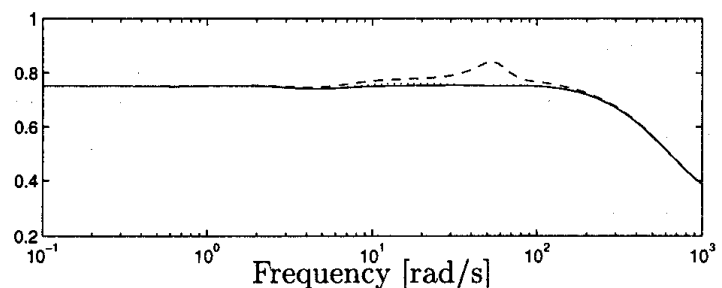

Figure 4: $\mu_{\Delta}\left(M_{\mathrm{Rp}}^{\star}\right)=\bar{\sigma}\left(D_{z} M_{\mathrm{RP}}^{\star} D_{w}^{-1}\right) \cdot(-)$ and $\bar{\sigma}\left(\tilde{D}_{z} M_{\mathrm{RP}}^{\star} \tilde{D}_{w}^{-1}\right)$ for $3 \mathrm{rd}(--)$ and 4 th order (..) DSE

\subsubsection{Results with WCU}

For the full IO set's optimal closed-loop $M_{\mathrm{RP}}^{\star}$, Fig. 5 depicts $\mu$ (its upper and lower bounds are virtually the same). The supremum $\alpha^{\star}:=\left\|M_{\mathrm{RP}}^{\star}\right\|_{\mu}=0.75$ is found at $\bar{\omega} \approx 33[\mathrm{rad} / \mathrm{s}]$. For the corresponding WCU data, the magnitude $\left(\left|\bar{\Delta}_{u}\right|=1 / \mu_{\Delta}\left(M_{\mathrm{RP}}^{\star}\right)\right)$ and phase are shown in Fig. 6. The all-pass WCU $\hat{\Delta}_{u}$ from dypert exactly equals the data $\bar{\Delta}_{u}$ at $\bar{\omega}$ and $\bar{\sigma}\left(\mathcal{F}_{u}\left(M_{\mathrm{RP}}^{\star}, \hat{\Delta}_{u}\right)\right)=\bar{\sigma}\left(\mathcal{F}_{u}\left(M_{\mathrm{RP}}^{\star}, \bar{\Delta}_{u}\right)\right)$ at $\bar{\omega}$. For other frequencies, where $\hat{\Delta}_{u}$ is not worst-case, the differences are large.

For three different $\gamma$ 's and hence three different $\tilde{\Delta}_{u}=$ $\frac{\alpha^{*}}{\gamma} \hat{\Delta}_{u},\left\|M_{\mathrm{wcu}}\right\|_{\infty}=\min _{K}\left\|\mathcal{F}_{l}\left(G_{\mathrm{wcU}}, K\right)\right\|_{\infty}$ is computed. The optimal values $\left\|M_{\mathrm{NP}}\right\|_{\infty}$ for Nominal Per-

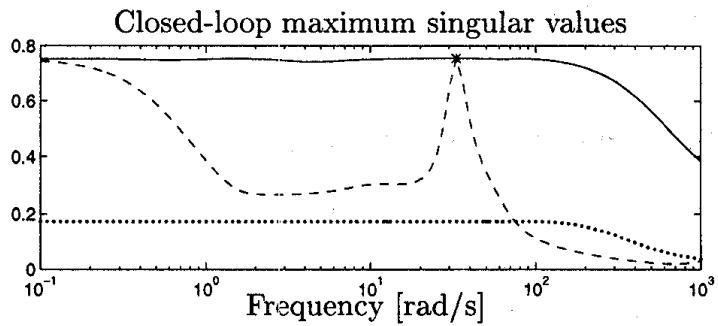

Figure 5: Closed-loop magnitudes with and without $\hat{\Delta}_{u}$ absorbed: $\mu_{\Delta}\left(M_{\mathrm{RP}}^{\star}\right)=\bar{\sigma}\left(\mathcal{F}_{u}\left(M_{\mathrm{RP}}^{\star}, \bar{\Delta}_{u}\right)\right)(-)$ and $\bar{\sigma}\left(\mathcal{F}_{u}\left(M_{\mathrm{RP}}^{\star}, \hat{\Delta}_{u}\right)\right)$ before $(--)$ and after $(. \cdot) \mathcal{H}_{\infty}$ optimization; * corresponds to the supremum of $\mu_{\Delta}\left(M_{\mathrm{RP}}^{\star}\right)$

formance (NP: no $\Delta_{u}$ ) are also printed. For the full IO set, the absorption of $\hat{\Delta}_{u}\left(=\tilde{\Delta}_{u}\right.$ for $\left.\gamma=\left\|M_{\mathrm{RP}}^{*}\right\|_{\mu}\right)$ into $G$ yields $\left\|M_{\mathrm{wcu}}^{\star}\right\|_{\infty}=0.17$. This is much smaller than $\left\|M_{\mathrm{RP}}^{\star}\right\|_{\mu}=0.75$, yielding a too optimistic indication of the achievable RP level. Figure 5 shows that $\mathcal{H}_{\infty}$ optimization for $G_{\mathrm{wCU}}^{\star}$ returns a closedloop magnitude which is considerably smaller than $\bar{\sigma}\left(\mathcal{F}_{u}\left(M_{\mathrm{RP}}^{\star}, \hat{\Delta}_{u}\right)\right)$ for most frequencies. This supports the earlier remark that, even for the full IO set itself, a WCU which is not worst-case for all frequencies may cause significant ineffectiveness.

Note that for $\gamma=1,\left\|M_{\mathrm{WCu}}\right\|_{\infty}$ is significantly smaller than $\left\|M_{\mathrm{RP}}\right\|_{\mu}$ for all IO sets, even for IO sets 2 and 6 with $\left\|M_{\mathrm{RP}}\right\|_{\mu}>1$. For $\gamma=0.8$ (i.e., for larger $\left.\tilde{\Delta}_{u}\right)$, IO sets 2 and 6 yield $\left\|M_{\text {wcu }}\right\|_{\infty}>0.8$, which is in line with their $\left\|M_{\mathrm{RP}}\right\|_{\mu}$ values. For IO set 8 , $\left\|M_{\mathrm{WCU}}\right\|_{\infty}<0.8$ despite the fact that $\left\|M_{\mathrm{RP}}\right\|_{\mu}>0.8$.

Another observation from Table 1 is that $\left\|M_{\mathrm{NP}}\right\|_{\infty}$ for IO sets 5,7 , and 9 may be slightly larger than $\left\|M_{\text {wcu }}\right\|_{\infty}$. Moreover, for 10 sets 3,7 , and 9 the optimal $\left\|M_{\mathrm{wcu}}\right\|_{\infty}$ may slightly decrease for an increasing $\mathcal{H}_{\infty}$ norm of $\tilde{\Delta}_{u}$; for IO set 2 , the decrease is large if $\gamma=\left\|M_{\mathrm{RP}}\right\|_{\mu}$ is used instead of $\gamma=0.8$. Apparently, if the WCU $\hat{\Delta}_{u}$ is not truly worst-case, absorbing it into the plant may have a positive effect on the plant dynamics. This leads to the counterintuitive phenomenon that "performance becomes better in the case of (larger) uncertainty." 

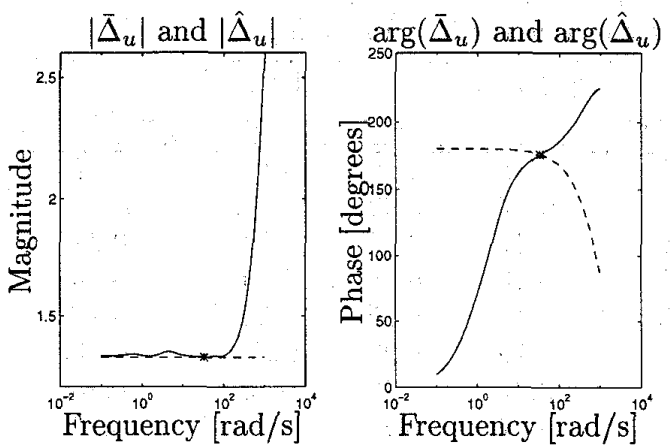

Figure 6: WCU data $\bar{\Delta}_{u}(-)$ for full IO set and allpass WCU representation $\hat{\Delta}_{u}(--) ; *$ corresponds to the supremum of $\mu_{\Delta}\left(M_{\mathrm{RP}}^{\star}\right)$

\subsection{IO selection results}

For $\gamma=1$ and $\gamma=0.8$, Table 2 summarizes the results for IO selection with the DSE (fourth order $\tilde{D}_{z}$ and $\tilde{D}_{w}$ ) and the WCU (first order $\tilde{\Delta}_{u}$ ), as well as the results from "suboptimal" DKI. In the latter case, DKI is stopped if $\left\|M_{\mathrm{RP}}\right\|_{\mu}<\gamma$ or if $\left\|M_{\mathrm{RP}}\right\|_{\mu}$ from subsequent iterations does not further decrease than 0.01 . Assuming the $D-K$ iteration results to be correct, they serve to assess the effectiveness of the new methods. The CPU times are normalized with respect to the (fastest) WCU method.

For $\gamma=1$, DKI rejects the 15 IO sets with the single $u_{1}$ and the two IO sets $y_{1} / u_{1} u_{2}, y_{1} / u_{2}$ with the single $y_{1}$. The DSE method rejects 19 IO sets, but $y_{3} / u_{1} u_{2}$ and $y_{3} / u_{2}$ can achieve $\left\|M_{\mathrm{RP}}\right\|_{\mu}=0.83$ and are incorrectly rejected. The WCU method accepts all 45 IO sets, so 17 are incorrectly accepted. For $\dot{\gamma}=0.8, y_{3} / u_{1} u_{2}$ and $y_{3} / u_{2}$ using the single $y_{3}$ should be rejected in addition to the 17 IO sets rejected for $\gamma=1$. The WCU method incorrectly accepts these two IO sets, while the DSE method incorrectly eliminates $y_{1} y_{3} / u_{1} u_{2}$ and $y_{1} y_{3} / u_{2}$ with $\left\|M_{\mathrm{RP}}\right\|_{\mu}=0.76$. The above clearly illustrates the sufficient character of the DSE method and the necessary character of the WCU method. The new methods may thus be ineffective, but combining them with DKI for IO sets accepted by WCU and rejected by DSE (here: four IO sets) would give an effective method.

Table 2 also shows that for this problem the WCU method is somewhat faster than the DSE method. This is due to the order of $G_{\text {WCU }}$ being smaller than the order of $G_{\mathrm{DSE}}$, which reduces the time needed for checking the $\mathcal{H}_{\infty}$ controller existence conditions. Both methods are significantly faster than DKI. However, it is emphasized that the CPU times depend on the particular example and on implementation aspects like the order of the $D$-scale approximations and the number of frequencies for DKI.
Table 2: Number of accepted 10 sets and relative CPU times

\begin{tabular}{|c|c|c|c|c|c|c|}
\hline \multirow[t]{2}{*}{$\gamma$} & \multirow{2}{*}{$\begin{array}{r}\text { DKI } \\
\text { Accepted }\end{array}$} & \multirow{2}{*}{$\mathrm{CPU}$} & \multirow{2}{*}{\multicolumn{2}{|c|}{ DSE }} & \multicolumn{2}{|c|}{ WCU } \\
\hline & & & & & ented & $\mathrm{CF}$ \\
\hline 0 & 28 & 53 & 26 & 1. & & \\
\hline 1.8 & 26 & 91 & 24 & 1.6 & 28 & 1 \\
\hline
\end{tabular}

\section{Discussion}

An example showed that the DSE method may incorrectly reject IO sets due to sufficiency, while the WCU method may incorrectly accept IO sets due to necessity. Compared to DKI, the efficiency of both methods was considerably better. IO selection by subsequent application of the WCU, DSE, and DKI methods is effective and possibly (for the example: certainly) more efficient than DKI alone.

Three topics for future research are suggested. First, it could be investigated if the effectiveness of the new methods could be predicted beforehand. More specifically, if conditions on the generalized plant could be developed under which applying a DSE or WCU is guaranteed to be more successful than checking without DSE $\left(\left\|M_{\mathrm{RP}}\right\|_{\infty}<\gamma\right)$ or without WCU $\left(\left\|M_{\mathrm{NP}}\right\|_{\infty}<\gamma\right)$. Second, procedures could be developed for generating or constructing a WCU that eliminates some of the ineffectiveness for the full IO set. Third, it should be investigated if the $\mu$ upper bound for mixed real/complex $\Delta[6]$ provides prospects for extending the current methods to deal with both dynamic and parametric uncertainties.

\section{References}

[1] G. J. Balas, J. C. Doyle, K. Glover, A. Packard, and R. Smith. $\mu$-Analysis and synthesis toolbox. The MathWorks, Natick, MA, 1995. version 3.0.

[2] P. Gahinet and P. Apkarian. A linear matrix inequality approach to $\mathcal{H}_{\infty}$ control. Int. J. Robust and Nonlinear Control, 4(1):421-448, 1994.

[3] M. van de Wal. Input output selection based on worst case uncertainty. Technical Report WFW 97.015, Fac. of Mechanical Engineering, Eindhoven University of Technology, 1997.

[4] M. van de Wal and B. de Jager. Selection of sensors and actuators for an active suspension control problem. In Proc. of the 1996 IEEE Int. Conf. Control Applications, pages 55-60, Dearborn, MI, 1996.

[5] M. van de Wal and B. de Jager. Selection of sensors and actuators based on a sufficient condition for robust performance. In Proc. of the European Control Conf., Brussels, Belgium, 1997.

[6] P. Young. Controller design with real parametric uncertainties. Int. J. Control, 65(3):469-509, 1996.

[7] K. Zhou, J. C. Doyle, and K. Glover. Robust and optimal control. Prentice-Hall, Upper Saddle River, NJ, 1996. 